\title{
Estimation de l'enthalpie de fusion de mélanges eutectiques de sels fondus utilisables pour le stockage thermique de l'énergie
}

\author{
J. P. Bros et M. Gaune-Escard
}

Laboratoire de Dynamique et Thermophysique des Fluides (*)
Université de Provence, Centre de St-Jérôme, rue Henri-Poincaré, 13397 Marseille Cedex 4, France

(Reçu le 5 juillet 1978, révisé le 11 octobre 1978, accepté le 16 octobre 1978)

\begin{abstract}
Résumé. - Nous avons envisagé l'utilisation de l'enthalpie de fusion de mélanges eutectiques de sels fondus pour le stockage thermique de l'énergie.

Un calcul thermodynamique simple a été établi : il permet d'évaluer l'enthalpie de fusion à partir de la connaissance des variations d'enthalpie des corps purs. Nous avons ainsi déterminé les enthalpies de fusion d'une quarantaine de mélanges eutectiques binaires et ternaires de sels fondus.
\end{abstract}

\begin{abstract}
The use of the enthalpy of fusion of eutectic molten salt mixtures has been considered for thermal energy storage.

A simple thermodynamic calculation has been stated : it enables to evaluate the enthalpy of fusion from the known enthalpy variations of pure substances. The enthalpies of fusion has been so determined for about fourty eutectic binary and ternary molten salt mixtures.
\end{abstract}

1. Position du problème. - Pour améliorer le rendement de certaines unités de production d'énergie centrales nucléaires - ou mettre en œuvre de nouvelles sources d'énergie - centrales solaires - un système de stockage de l'énergie est nécessaire entre le centre producteur et les utilisateurs [1] ; cette énergie, ainsi stockée, pourrait alors être cédée soit en période de forte demande (heures de pointe), soit en période de faible production (périodes sans soleil). Il existe de nombreux projets pour stocker l'énergie; nous nous sommes plus particulièrement intéressés aux aspects fondamentaux du stockage thermique.

Le premier principe de la thermodynamique permet d'évaluer la chaleur emmagasinée, à pression constante, lors de l'élévation de la température $\left(T_{1} \rightarrow T_{2}\right)$ d'une masse $\mathrm{m}$ de substance de masse molaire $M$ :

$$
\begin{aligned}
\Delta H & =H_{T_{2}}-H_{T_{1}}=\frac{m}{M}\left[\int_{T_{1}}^{T_{\text {fus. }}} C_{\mathrm{p}(\mathrm{s})} \mathrm{d} T+\right. \\
& \left.+\Delta H_{\text {fus. }}+\int_{T_{\text {fus. }}}^{T_{2}} C_{\mathrm{p}(1)} \mathrm{d} T\right]
\end{aligned}
$$

relation dans laquelle $C_{\mathrm{p}(\mathrm{s})}$ et $C_{\mathrm{p}(1)}$ sont respectivement les capacités calorifiques molaires à pression constante à l'état solide et liquide, et $\Delta H_{\text {fus. }}$ l'enthalpie molaire de fusion à la température $T_{\text {fus. }}$. Lorsque le matériau choisi présente d'autres changements de

$\left({ }^{*}\right)$ Associé au C.N.R.S. phase, une relation analogue permet d'en tenir compte. Lors du refroidissement, la même quantité de chaleur est restituée.

Du point de vue purement thermodynamique, le matériau sera d'autant plus intéressant pour le stockage thermique de l'énergie que cet incrément enthalpique $\Delta H$ sera élevé. Le choix de ce matériau est guidé, non seulement par des conditions physiques évidentes (faible viscosité, bonne conductibilité thermique, tension de vapeur négligeable, variation de volume à fusion connue...), mais aussi par :

- des critères chimiques [2] : le fluide doit être peu corrosif et stable dans les conditions d'emploi (température et temps),

— des critères économiques [3] : le prix de revient aussi faible que possible,

— des critères écologiques relatifs à la sécurité de l'environnement,

Ces derniers critères sont en général plus difficiles à apprécier.

Pour le stockage thermique à haute température $(T>600 \mathrm{~K})$, nous ne nous sommes intéressés qu'à des corps ou des mélanges de corps inorganiques à l'état condensé ; en effet, les métaux, les composés ioniques ou leurs mélanges sont beaucoup plus stables que les produits organiques. Dans ce qui suit, nous avons limité notre étude à l'évaluation de l'enthalpie de fusion de composés ioniques. 
Aux conditions énoncées ci-dessus s'ajoute celle imposée par la température d'utilisation ultérieure (rendement maximum de la machine thermique placée en aval du système de stockage) : la température de cristallisation du matériau de stockage doit en être la plus voisine possible. Le choix de sels fondus purs étant ainsi restreint, nous avons été conduits à envisager également l'emploi de mélanges eutectiques binaires ou ternaires de sels fondus; ceux-ci sont nombreux et les chances de trouver une température de fusion aussi voisine que possible de la température optimale d'utilisation sont alors plus grandes.

2. Evaluation de l'enthalpie de fusion de mélanges eutectiques. - L'enthalpie de fusion d'un mélange eutectique peut être obtenue soit par mesure directe, soit par un calcul thermodynamique formel.

Par analyse enthalpique différentielle [4-6] ou par calorimétrie de chute [7], l'enthalpie de fusion peut être mesurée avec une bonne précision. Cette procédure expérimentale est en général très longue ce qui explique le peu de données disponibles dans la littérature.

Aussi avons-nous évalué les enthalpies de fusion de plusieurs mélanges eutectiques binaires et ternaires à l'aide de la loi de Hess.

Considérons un diagramme d'équilibre des phases (Fig. 1a) présentant un point eutectique $\mathrm{E}$ et deux solutions solides extrémales $\alpha$ et $\beta^{\circ}$. L'énergie stockée donnée par l'équation (1) correspond, sur ce diagramme de phase, au chemin NM. Il est possible aussi de passer de l'état $\mathrm{N}$ à l'état $\mathrm{M}$ en suivant un autre chemin thermodynamique (Fig. $1 b$ ).

Les variations de l'enthalpie relatives aux deux chemins symbolisés par (I) et (II + III + IV) sont égales, les états initial et final étant identiques.

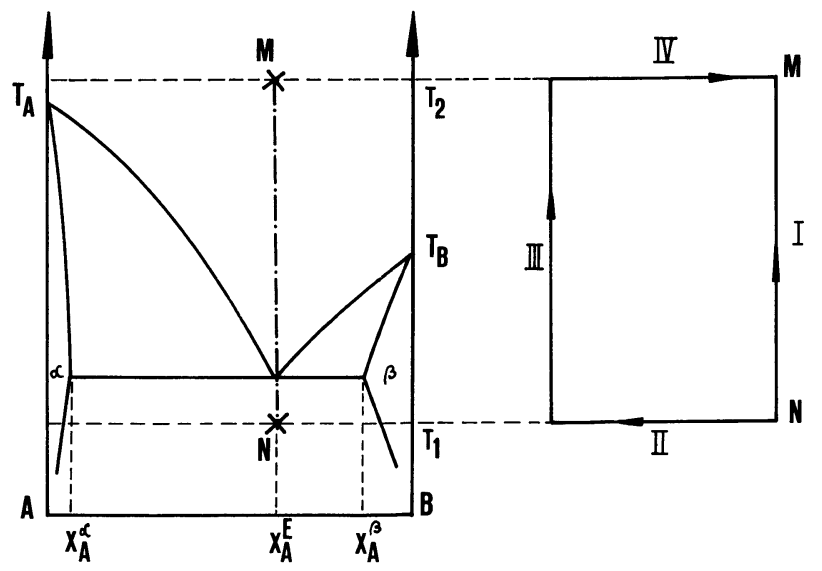

a)

b)

Fig. 1a. - Diagramme d'équilibre des phases binaires présentant un point eutectique et des solutions solides extrémales $\alpha$ et $\beta$.

[Binary equilibrium phase diagram with eutectic point and limiting solid solutions $\alpha$ and $\beta$.]

Fig. 1b. - Cycle thermodynamique.

[Thermodynamic cycle.]
Nous donnons ci-dessous ces variations d'enthalpie ramenées à une mole

$$
\begin{aligned}
& \Delta H_{(\mathrm{I})}=\int_{T_{1}}^{T_{\mathrm{E}}} C_{\mathrm{p}(\mathrm{s})}^{\mathrm{E}} \mathrm{d} T+\Delta H_{\mathrm{fus}}^{\mathrm{E}}+\int_{T_{\mathrm{E}}}^{T_{2}} C_{\mathrm{p}(1)}^{\mathrm{E}} \mathrm{d} T \\
& \Delta H_{\text {(II) }}=\left(\Delta H_{\mathbf{M}}\right)_{X_{\mathbf{A}}=X_{\mathbf{A}}^{\mathbf{E}}} \\
& \Delta H_{(\mathrm{III})}=\left[\int_{T_{1}}^{T_{\mathrm{A}}} C_{\mathrm{p}(\mathrm{s})}^{\mathrm{A}} \mathrm{d} T+\Delta H_{\mathrm{fus} .}^{\mathrm{A}}+\right. \\
& \left.+\int_{T_{\mathrm{A}}}^{T_{2}} C_{\mathrm{p}(1)}^{\mathrm{A}} \mathrm{d} T\right] X_{\mathrm{A}}^{\mathrm{E}} \\
& +\left[\int_{T_{1}}^{T_{\mathrm{B}}} C_{\mathrm{p}(\mathrm{s})}^{\mathrm{B}} \mathrm{d} T+\Delta H_{\text {fus. }}^{\mathrm{B}}+\int_{T_{\mathrm{B}}}^{T_{2}} C_{\mathrm{p}(1)}^{\mathrm{B}} \mathrm{d} T\right] X_{\mathrm{B}}^{\mathrm{E}} \\
& \Delta H_{(\mathrm{IV})}=\frac{X_{\mathrm{A}}^{\mathrm{E}}-X_{\mathrm{A}}^{\beta}}{X_{\mathrm{A}}^{\alpha}-X_{\mathrm{A}}^{\beta}} \cdot \Delta H_{\text {form. }}^{\alpha}+ \\
& +\frac{X_{\mathrm{A}}^{\alpha}-X_{\mathrm{A}}^{\mathrm{E}}}{X_{\mathrm{A}}^{\alpha}-X_{\mathrm{A}}^{\beta}} \cdot \Delta H_{\text {form. }}^{\beta} \equiv \Delta H_{\text {form.(s) }}^{\mathrm{E}} .
\end{aligned}
$$

L'expression précédente de $\Delta H(\mathrm{IV})$, qui est l'enthalpie de formation du mélange eutectique solide, a été obtenue par application d'une loi linéaire de mélange (règle du levier); $\Delta H_{\mathrm{form}}^{\alpha}$ et $\Delta H_{\mathrm{form}}^{\beta}$ sont les enthalpies de formation des phases $\alpha$ et $\beta$ respectivement, de composition $X_{\mathrm{A}}^{\alpha}$ et $X_{\mathrm{A}}^{\beta}$.

De l'ensemble de ces relations, et en respectant l'égalité ci-dessus énoncée, on obtient l'expression de l'enthalpie de fusion de mélange eutectique $\Delta H_{\text {fus. }}^{\mathrm{E}}$ :

$$
\begin{aligned}
& \Delta H_{\text {fus. }}^{\mathrm{E}}=X_{\mathrm{A}}^{\mathrm{E}} \Delta H_{\text {fus. }}^{\mathrm{A}}+X_{\mathrm{B}}^{\mathrm{E}} \Delta H_{\text {fus. }}^{\mathrm{B}}+ \\
& \quad+\left(\Delta H_{\mathrm{M}}\right)_{X_{\mathrm{A}}=X_{\mathrm{A}}^{\mathrm{E}}}-\Delta H_{\text {form.(s) }}^{\mathrm{E}}+U
\end{aligned}
$$

avec

$$
\begin{aligned}
U= & {\left[\int_{T_{1}}^{T_{\mathrm{A}}} C_{\mathrm{p}(\mathrm{s})}^{\mathrm{A}} \mathrm{d} T+\int_{T_{\mathrm{A}}}^{T_{2}} C_{\mathrm{p}(1)}^{\mathrm{A}} \mathrm{d} T\right] X_{\mathrm{A}}^{\mathrm{E}} } \\
& +\left[\int_{T_{1}}^{T_{\mathrm{B}}} C_{\mathrm{p}(\mathrm{s})}^{\mathrm{B}} \mathrm{d} T+\int_{T_{\mathrm{B}}}^{T_{2}} C_{\mathrm{p}(1)}^{\mathrm{B}} \mathrm{d} T\right] X_{\mathrm{B}}^{\mathrm{E}} \\
& -\left[\int_{T_{1}}^{T_{\mathrm{E}}} C_{\mathrm{p}(\mathrm{s})}^{\mathrm{E}} \mathrm{d} T+\int_{T_{\mathrm{E}}}^{T_{2}} C_{\mathrm{p}(1)}^{\mathrm{E}} \mathrm{d} T\right]
\end{aligned}
$$

Des hypothèses, rendues indispensables par le faible nombre de données expérimentales relatives aux mélanges, sont nécessaires pour l'application de la formule précédente. Nous avons admis comme beaucoup d'auteurs :

- que les solutions solides sont idéales (ceci n'est valable que pour des solutions solides peu étendues),

- que la loi sur l'additivité des chaleurs spécifiques (loi de Kopp et Neumann) est vérifiée dans tout le domaine de température ce qui conduit à négliger les termes $\Delta H_{\text {form.(s) }}^{\mathrm{E}}$ et $U$ dans la relation précédente. 
Tableau I. - Enthalpies de fusion de sels fondus purs $(\mathrm{kcal}=4,184 \mathrm{~kJ})$.

[Enthalpies of fusion of pure molten salts $(\mathrm{kcal}=4.184 \mathrm{~kJ})$.]

\begin{tabular}{|c|c|c|c|c|c|c|}
\hline Sel & $\begin{array}{c}T_{\text {fus. }} \\
\mathrm{K}\end{array}$ & 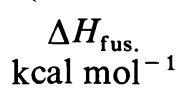 & $\begin{array}{c}M \\
\mathrm{~g}\end{array}$ & $\begin{array}{c}\Delta H_{\text {fus. }} \\
\mathrm{cal} / \mathrm{g}\end{array}$ & $\begin{array}{l}d\left({ }^{*}\right) \text { (liq.) } \\
\mathrm{g} / \mathrm{cm}^{3}\end{array}$ & $\begin{array}{l}\Delta H_{\text {fus. }} \\
\mathrm{cal} / \mathrm{cm}^{3}\end{array}$ \\
\hline - & - & - & - & - & - & - \\
\hline $\mathrm{LiF}$ & 1121 & 6,47 & 25,94 & 249 & 1,80 & 449 \\
\hline $\mathrm{LiCl}$ & 883 & 4,76 & 42,39 & 112 & 1,50 & 168 \\
\hline $\mathrm{LiBr}$ & 823 & 4,22 & 86,85 & 49 & 2,51 & 123 \\
\hline LiI & 742 & 3,50 & 133,84 & 26 & 3,12 & 81 \\
\hline $\mathrm{NaF}$ & 1268 & 8,03 & 41,99 & 191 & 1,95 & 372 \\
\hline $\mathrm{NaCl}$ & 1073 & 6,69 & 58,44 & 115 & 1,55 & 178 \\
\hline $\mathrm{NaBr}$ & 1020 & 6,24 & 102,90 & 61 & 2,33 & 142 \\
\hline $\mathrm{NaI}$ & 933 & 5,64 & 149,89 & 55 & 1,87 & 103 \\
\hline $\mathrm{KF}$ & 1131 & 6,75 & 58,10 & 116 & 1,91 & 221 \\
\hline $\mathrm{KCl}$ & 1043 & 6,34 & 74,56 & 85 & 1,53 & 130 \\
\hline $\mathrm{KBr}$ & 1007 & 6,10 & 119,01 & 51 & 2,14 & 109 \\
\hline KI & 954 & 5,74 & 166,01 & 35 & 2,47 & 84 \\
\hline $\mathrm{RBCl}$ & 995 & 5,67 & 120,92 & 47 & 2,23 & 105 \\
\hline $\mathrm{RbBr}$ & 965 & 5,57 & 165,38 & 34 & 2,68 & 91 \\
\hline RBI & 920 & 5,27 & 212,37 & 25 & 2,88 & 72 \\
\hline $\mathrm{CsF}$ & 976 & 5,19 & 151,90 & 34 & 3,68 & 125 \\
\hline $\mathrm{CsCl}$ & 918 & 4,84 & 168,36 & 29 & 2,76 & 80 \\
\hline $\mathrm{CsBr}$ & 909 & 5,64 & 272,81 & 22 & 3,77 & 83 \\
\hline CsI & 899 & 5,64 & 259,81 & 22 & 3,14 & 69 \\
\hline $\mathrm{CaF}_{2}$ & 1684 & 7,1 & 78,08 & 91 & 2,95 & 268 \\
\hline $\mathrm{CaCl}_{2}$ & 1045 & 6,78 & 110,99 & 61 & 2,08 & 127 \\
\hline $\mathrm{CaBr}_{2}$ & 1015 & 6,95 & 199,90 & 35 & 3,11 & 109 \\
\hline $\mathrm{CaI}_{2}$ & 1052 & 10,0 & 293,89 & 34 & 3,11 & 109 \\
\hline $\mathrm{BaF}_{2}$ & 1593 & 6,96 & 175,34 & 40 & 3,98 & 158 \\
\hline $\mathrm{BaCl}(* *)(\alpha, \beta)$ & 1235 & 8,03 & 208,25 & 39 & 3,16 & 122 \\
\hline $\mathrm{BaBr}_{2}$ & 1150 & 7,63 & 297,16 & 26 & 4,00 & 104 \\
\hline $\mathrm{BaI}_{2}$ & 1013 & 6,34 & 391,15 & 16 & 4,00 & 104 \\
\hline $\mathrm{SrF}_{2}$ & 1673 & 4,40 & 125,62 & 35 & 3,43 & 120 \\
\hline $\operatorname{SrCl}(* *)\left(\alpha, \beta^{2}\right)$ & 1146 & 5,53 & 158,53 & 35 & 2,72 & 95 \\
\hline $\operatorname{SrBr}\left({ }^{* *}\right)\left(\alpha, \beta^{2}\right)$ & 920 & 7,90 & 247,44 & 32 & 3,79 & $121-$ \\
\hline $\mathrm{SrI}_{2}$ & 788 & 4,70 & 341,43 & 14 & 3,79 & 121 \\
\hline $\mathrm{MgF}_{2}$ & 1536 & 13,90 & 62,31 & 223 & 2,40 & 535 \\
\hline $\mathrm{MgCl}_{2}$ & 981 & 10,30 & 95,22 & 108 & 1,69 & 182 \\
\hline $\mathrm{MgBr}_{2}$ & 987 & 8,85 & 184,13 & 48 & 2,63 & 126 \\
\hline $\mathrm{Li}_{2} \mathrm{CO}_{3}$ & 891 & 10,70 & 73,89 & 145 & 1,83 & 265 \\
\hline $\mathrm{Na}_{2} \mathrm{CO}_{3}$ & 1131 & 6,70 & 105,99 & 63 & 1,97 & 125 \\
\hline $\mathrm{K}_{2} \mathrm{CO}_{3}$ & 1172 & 6,60 & 138,21 & 48 & 1,90 & 91 \\
\hline $\mathrm{LiNO}_{3}$ & 526 & 6,39 & 68,95 & 93 & 1,78 & 165 \\
\hline $\mathrm{NaNO}_{3}$ & 580 & 3,60 & 85,01 & 42 & 1,92 & 81 \\
\hline $\mathrm{KNO}_{3}$ & 607 & 2,30 & 101,1 & 23 & 1,87 & 43 \\
\hline $\mathrm{RbNO}_{3}\left({ }^{* *}\right)(\alpha, \beta, \gamma, \delta)$ & 583 & 3,03 & 147,49 & 21 & 2,49 & 51 \\
\hline $\mathrm{CsNO}_{3}\left({ }^{* *}\right)(\alpha, \beta)$ & 679 & 4,26 & 194,92 & 22 & 2,83 & 62 \\
\hline $\mathrm{NaOH}(* *)(\alpha, \beta)$ & 593 & 3,04 & 40,00 & 76 & 1,79 & 136 \\
\hline $\mathrm{KOH}$ & 673 & 1,83 & 56,11 & 33 & 1,70 & 56 \\
\hline $\mathrm{Li}_{2} \mathrm{SO}_{4}$ & 1132 & 1,98 & 109,95 & 18 & 2,00 & 36 \\
\hline $\mathrm{Na}_{2} \mathrm{SO}_{4}$ & 1157 & 5,67 & 142,05 & 40 & 2,07 & 83 \\
\hline $\mathrm{K}_{2} \mathrm{SO}_{4}{ }_{4}$ & 1349 & 8,76 & 174,27 & 50 & 1,94 & 98 \\
\hline
\end{tabular}

(**) Lorsque des transitions à l'état solide se produisent à des températures peu inférieures à la température de fusion, la valeur de l'enthalpie de fusion a été calculée en tenant compte de ces enthalpies de transition.

$[(* *)$ When solid state transformations occur at temperature not too far from the melting temperature, the values of enthalpies of fusion have been calculated taking into account these enthalpies of transition.] 
Ce raisonnement peut être étendu, sans plus de difficultés, à un mélange eutectique à plus de deux constituants.

L'évaluation de l'enthalpie de fusion du mélange eutectique $\Delta H_{\text {fus. nécessite donc la connaissance des }}^{\mathrm{E}}$ enthalpies de fusion de chacun des corps purs le constituant, données généralement disponibles, et de l'enthalpie de mélange à la concentration eutecti-

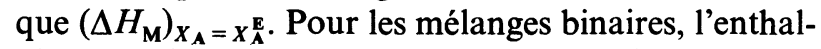
pie de mélange peut être obtenue soit directement à partir de l'expérience [8], soit indirectement à partir d'un calcul nécessitant une bonne connaissance du diagramme de phase [9].

Pour les mélanges ternaires, les résultats expérimentaux étant pratiquement inexistants, nous avons utilisé un modèle [10] permettant le calcul de l'enthalpie de mélange ternaire à partir de celles relatives aux trois systèmes binaires limitrophes.

3. Applications. - Nous rappelons (tableau I) les enthalpies de fusion de plusieurs sels purs. Ces grandeurs ont été données en $\mathrm{kcal} . \mathrm{mol}^{-1} \mathrm{et}$, pour des raisons pratiques (comparaison à masse ou volume identique), en cal $/ \mathrm{g}$ et $\mathrm{cal} / \mathrm{cm}^{3}$ en faisant intervenir les masses molaires et les densités à l'état liquide.

Le tableau II regroupe les résultats obtenus à partir du calcul précédent pour une trentaine de mélanges eutectiques binaires de sels fondus.

Le tableau III indique les résultats obtenus de la même manière pour onze mélanges eutectiques ternaires de sels fondus.

Les données figurant dans les tableaux précédents ont été rassemblées ou calculées à partir de divers ouvrages de synthèse ou de compilation [11-20].

La lecture de ces tableaux appelle quelques remarques :

- pour une température de stockage fixée, plusieurs mélanges eutectiques pourraient être utilisables (Tableau II),

- les sels qui présentent les enthalpies de fusion les plus grandes ont généralement les températures de fusion les plus élevées (Tableau I).

Dans ces conditions, toutes choses étant égales par ailleurs, est-il thermodynamiquement plus inté-

Tableau II. - Enthalpies de fusion de mélanges eutectiques binaires de sels fondus ( $\mathrm{kcal}=4,184 \mathrm{~kJ})$.

[Enthalpies of fusion of binary eutectic molten salt mixtures $(\mathrm{kcal}=4.184 \mathrm{~kJ})$.]

\begin{tabular}{|c|c|c|c|c|c|c|c|c|c|c|c|c|c|}
\hline Système A-B & $\begin{array}{c}\Delta H_{\mathrm{F}}(\mathrm{A}) \\
\mathrm{kcal} \mathrm{mol}^{-1}\end{array}$ & $\begin{array}{c}\Delta H_{\mathrm{F}}(\mathrm{B}) \\
\mathrm{kcal} \mathrm{mol}^{-1}\end{array}$ & $\begin{array}{c}\left(\Delta H_{\mathrm{M}}^{\mathrm{E}}\right)_{X_{\mathrm{A}}^{\mathrm{E}}} \\
\mathrm{kcal} \mathrm{mol}^{-1}\end{array}$ & $X_{\mathbf{A}}^{\mathbf{E}}$ & $\begin{array}{c}\Delta H_{\mathrm{fus}}^{\mathrm{E}} \\
\mathrm{kcal} \mathrm{mol}^{-1}\end{array}$ & $\begin{array}{c}M(\mathrm{E}) \\
\text { masse } \\
\text { molaire }\end{array}$ & $\begin{array}{c}\Delta H_{\mathrm{fus}}^{\mathrm{E}} \\
\mathrm{cal} / \mathrm{g}\end{array}$ & $\begin{array}{c}d\left({ }^{*}\right) \text { (liq.) } \\
\mathrm{g} / \mathrm{cm}^{3}\end{array}$ & $\begin{array}{c}\Delta H_{\text {fus. }} \\
\mathrm{cal} / \mathrm{cm}^{3}\end{array}$ & $\begin{array}{r}T_{\mathrm{A}} \\
(\mathrm{K})\end{array}$ & $\begin{array}{c}T_{\mathrm{B}} \\
(\mathrm{K})\end{array}$ & $\begin{array}{c}T_{\mathrm{E}} \\
(\mathrm{K})\end{array}$ & $\begin{array}{c}\varepsilon \\
(\mathrm{K})\end{array}$ \\
\hline $\mathrm{BaCl}_{2}-\mathrm{CaCl}_{2}$ & 8,0 & 6,78 & $-2,06$ & 0,34 & 5,14 & 144,06 & 35,68 & & & 1233 & $1045 \mid$ & 873 & 119 \\
\hline $\mathrm{BaCl}_{2}-\mathrm{KCl}$ & 8,0 & 6,34 & $-0,38$ & 0,26 & $5,40(\exp )$ & 109,19 & 53,36 & 2,06 & 110 & 1233 & 1043 & 938 & 77 \\
\hline $\mathrm{BaCl}_{2}-\mathrm{KCl}$ & 8,0 & 6,34 & $-0,49$ & 0,43 & $5,55(\exp )$ & 131,91 & 42,07 & 2,47 & 104 & 1233 & 1043 & 928 & 103 \\
\hline $\mathrm{BaCl}_{2}-\mathrm{MgCl}_{2}$ & 8,0 & 10,3 & $-0,55$ & 0,64 & 8,28 & 167,56 & 49,42 & 2,49 & 123 & 1233 & 987 & 833 & 128 \\
\hline $\mathrm{BaCl}_{2}-\mathrm{NaCl}$ & 8,0 & 6,69 & $\sim 0$ & 0,39 & 7,20 & 116,87 & 61,61 & 2,54 & 157 & 1233 & $\left|\begin{array}{ll}1 & 073\end{array}\right|$ & 933 & 105 \\
\hline $\mathrm{CaCl}_{2}-\mathrm{CsCl}$ & 6,78 & 4,84 & $-1,1$ & 0,11 & 3,95 & 162,05 & 24,38 & 2,02 & 49 & 1045 & 918 & 883 & 19 \\
\hline $\mathrm{CaCl}_{2}-\mathrm{CsCl}$ & 6,78 & 4,84 & $-0,98$ & 0,89 & 5,59 & 117,30 & 47,66 & 2,35 & 112 & 1045 & 918 & 978 & 1 \\
\hline $\mathrm{CaCl}_{2}-\mathrm{KCl}$ & 6,78 & 6,34 & $-1,82$ & 0,25 & 4,63 & 83,67 & 55,34 & 2,00 & 111 & 1045 & $1-043$ & 888 & 59 \\
\hline $\mathrm{CaCl}_{2}-\mathrm{KCl}$ & 6,78 & 6,34 & $-1,65$ & 0,75 & 5,02 & 101,88 & 49,27 & 1,77 & 87 & 1045 & 1043 & 923 & 45 \\
\hline $\mathrm{CaCl}_{2}-\mathrm{LiCl}$ & 6,78 & 4,76 & $-0,11$ & 0,38 & 5,42 & 68,46 & 79,17 & 1,83 & 145 & 1045 & 883 & 769 & 92 \\
\hline $\mathrm{CaCl}_{2}-\mathrm{MgCl}_{2}$ & 6,78 & 10,3 & $+0,20$ & 0,47 & 8,85 & 102,63 & 86,23 & 2,76 & 238 & 1045 & 987 & 883 & 66 \\
\hline $\mathrm{CaCl}_{2}-\mathrm{NaCl}$ & 6,78 & 6,69 & $-0,78$ & 0,48 & 5,97 & 83,66 & 71 & 1,85 & 131 & 1045 & $\begin{array}{ll}1 & 073\end{array} \mid$ & 773 & 130 \\
\hline $\mathrm{CsCl}-\mathrm{NaCl}$ & 4,84 & 6,69 & $-0,22$ & 0,65 & 5,27 & 129,89 & 40,6 & 2,36 & 96 & 918 & 1073 & 763 & 106 \\
\hline $\mathrm{KCl}-\mathrm{LiCl}$ & 6,34 & 4,76 & $-1,04$ & 0,416 & 4,38 & 55,77 & 78,54 & 1,64 & 128,5 & 1043 & 883 & 740 & 109 \\
\hline $\mathrm{LiCl}-\mathrm{RbCl}$ & 4,76 & 5,67 & $-1,20$ & 0,56 & 3,96 & 76,94 & 51,47 & 2,25 & 116 & 883 & 995 & 583 & 175 \\
\hline $\mathrm{NaCl}-\mathrm{RbCl}$ & 6,69 & 5,67 & $-0,20$ & 0,46 & 5,94 & 92,18 & 64,44 & 2,06 & 133 & $\left|\begin{array}{ll}1 & 073\end{array}\right|$ & 995 & 813 & 110 \\
\hline $\mathrm{NaCl}-\mathrm{SrCl}_{2}$ & 6,69 & 5,53 & $-0,23$ & 0,49 & 5,87 & 109,49 & 53,61 & - & - & 1073 & 1146 & 838 & 136 \\
\hline $\mathrm{BaBr}_{2}-\mathrm{NaBr}$ & 7,63 & 6,24 & $+0,04$ & 0,41 & 6,85 & 182,55 & 37,52 & - & - & 1130 & $\begin{array}{ll}1 & 020\end{array}$ & 873 & 98 \\
\hline CsBr-LiBr & 5,64 & 4,22 & $-1,22$ & 0,37 & 3,53 & 133,46 & 26,45 & 2,69 & 71,15 & 909 & 823 & 535 & 154 \\
\hline $\mathrm{CsBr}-\mathrm{NaBr}$ & 5,64 & 6,24 & $-0,27$ & 0,38 & 5,74 & 144,67 & 39,68 & - & - & 909 & 1020 & 733 & 109 \\
\hline $\mathrm{KBr}-\mathrm{LiBr}$ & 6,10 & 4,22 & $-0,81$ & 0,40 & 4,16 & 99,71 & 41,72 & 2,53 & 105,55 & $\begin{array}{lll}1 & 0 & 07\end{array}$ & 823 & 603 & 150 \\
\hline $\mathrm{LiBr}-\mathrm{NaBr}$ & 4,22 & 6,24 & $-0,16$ & 0,80 & 4,46 & 90,06 & 49,52 & 2,55 & 126,28 & 823 & 1020 & 780 & 70 \\
\hline LiBr-RbBr & 4,22 & 5,57 & $-1,03$ & 0,60 & 3,73 & 118,26 & 31,54 & $(0,49)$ & $(15,45)$ & 823 & 965 & 532 & 174 \\
\hline $\mathrm{NaBr}-\mathrm{RbBr}$ & 6,24 & 5,57 & $-0,22$ & 0,48 & 5,67 & 135,39 & 41,89 & 2,57 & 107,6 & 1020 & 965 & 768 & 112 \\
\hline $\mathrm{CaF}_{2}-\mathrm{LiF}$ & 8,2 & 6,47 & $-0,2$ & 0,2 & 6,62 & 36,37 & 182 & 2,05 & 373 & 1684 & $\begin{array}{lll}1 & 121\end{array}$ & 1042 & 115 \\
\hline $\mathrm{CaF}_{2}-\mathrm{NaF}$ & 8,2 & 8,03 & $-1,02$ & 0,32 & 7,06 & 53,54 & 132 & 2,27 & 286 & 1684 & 1268 & 1080 & 172 \\
\hline KF-LiF & 6,75 & 6,47 & $-1,17$ & 0,50 & 5,44 & 42,02 & 129,5 & 1,81 & 234 & $\begin{array}{lll}1 & 131\end{array}$ & $\begin{array}{ll}1 & 121\end{array}$ & 763 & 182 \\
\hline KF-NaF & 6,75 & 8,03 & $-0,22$ & 0,60 & 7,04 & 51,66 & 136,3 & 2,05 & 279 & $\begin{array}{lll}1 & 131\end{array}$ & 1268 & 973 & 109 \\
\hline $\mathrm{LiF}-\mathrm{NaF}$ & 6,47 & 8,03 & $-0,48$ & 0,60 & 6,61 & 32,36 & 204,3 & 2,17 & 444,2 & $\begin{array}{lll}1 & 121\end{array}$ & 1268 & 925 & 129 \\
\hline LiF-RbF & 6,47 & 6,15 & $-1,2$ & 0,44 & 5,09 & 69,92 & 72,80 & 2,84 & 206,75 & $\begin{array}{lll}1 & 121\end{array}$ & 1063 & 748 & 170 \\
\hline LiCl-LiF & 4,76 & 6,47 & $-0,19$ & 0,71 & 5,07 & 37,6 & 134,84 & 1,55 & 209,1 & 883 & 1121 & 758 & 100 \\
\hline
\end{tabular}

(*) Densité du mélange liquide au point de fusion.

$\left[\left(^{*}\right)\right.$ Liquid density at melting point.] 
Tableau III. - Enthalpies de fusion de mélanges eutectiques ternaires de sels fondus ( $\mathrm{kcal}=4,184 \mathrm{~kJ})$.

[Enthalpies of fusion of ternary eutectic molten salt mixtures $(\mathrm{kcal}=4.184 \mathrm{~kJ})$.]

\begin{tabular}{|c|c|c|c|c|c|c|c|c|c|c|}
\hline $\begin{array}{c}\text { Système 1-2-3 } \\
x_{1}-x_{2}-x_{3}(\mathrm{~mol} \%)\end{array}$ & $\begin{array}{c}\Delta H_{\mathrm{F}}(1) \\
\mathrm{kcal} \mathrm{mol}^{-1}\end{array}$ & $\begin{array}{c}\Delta H_{\mathrm{F}}(2) \\
\mathrm{kcal} \mathrm{mol}^{-1}\end{array}$ & $\begin{array}{c}\Delta H_{\mathrm{F}}(3) \\
\mathrm{kcal} \mathrm{mol}^{-1}\end{array}$ & $\begin{array}{c}\Delta H_{\mathrm{M}} \\
\mathrm{kcal} \mathrm{mol}^{-1}\end{array}$ & $\begin{array}{l}d\left({ }^{*}\right) \\
\text { (liq.) } \\
\mathrm{g} / \mathrm{cm}^{3}\end{array}$ & $\begin{array}{l}T_{\mathrm{E}} \\
\mathrm{K}\end{array}$ & $\begin{array}{c}\bar{M} \\
\mathrm{~g}\end{array}$ & $\underset{\mathrm{kcal} \mathrm{mol}^{-1}}{\Delta H_{\text {fus.(E) }}}$ & $\begin{array}{c}\Delta H_{\text {fus.(E) }} \\
\mathrm{cal} / \mathrm{g}\end{array}$ & $\begin{array}{l}\Delta H_{\text {fus.(E) }} \\
\mathrm{cal} / \mathrm{cm}^{3}\end{array}$ \\
\hline $\begin{array}{l}\mathrm{KCl}-\mathrm{NaCl}-\mathrm{MgCl}_{2} \\
12,9 \quad 15,5 \quad 71,6\end{array}$ & 6,34 & 6,69 & 10,3 & $-2,20$ & 1,63 & 658 & 84 & 7,03 & 83,7 & 136,4 \\
\hline $\begin{array}{l}\mathrm{K}_{2} \mathrm{CO}_{3}-\mathrm{Li}_{2} \mathrm{CO}_{3}-\mathrm{Na}_{2} \mathrm{CO}_{3} \\
25 \quad 43,5 \quad 31,5\end{array}$ & 6,6 & 10,7 & 6,7 & $-2,81$ & 1,90 & 670 & 100,1 & 5,60 & 55,9 & 106,3 \\
\hline $\begin{array}{l}\mathrm{NaCl}-\mathrm{BaCl}_{2}-\mathrm{MgCl}_{2} \\
46 \quad 14,5 \quad 39,5\end{array}$ & 6,69 & 8,0 & 10,3 & $-2,20$ & 2,12 & 691 & 94,7 & 6,11 & 64,5 & 136,8 \\
\hline $\begin{array}{l}\text { NaF-KF - } \mathrm{LiF} \\
11,5 \quad 42,0 \quad 46,5\end{array}$ & 8,03 & 6,50 & 6,47 & $-1,42$ & 1,90 & 727 & 41,3 & 5,24 & 126,9 & 241,1 \\
\hline $\begin{array}{l}\mathrm{KCl}-\mathrm{CaCl}_{2}-\mathrm{MgCl}_{2} \\
30,922,2 \quad 46,9\end{array}$ & 6,34 & 6,78 & 10,3 & $-5,10$ & 1,74 & 760 & 92,4 & 3,20 & 34,6 & 60,3 \\
\hline $\begin{array}{l}\mathrm{KCl}-\mathrm{NaCl}-\mathrm{CaCl}_{2} \\
42,5 \quad 51,7 \quad 5,0\end{array}$ & 6,34 & 6,69 & 6,78 & $-1,40$ & 1,90 & 777 & 86,6 & 5,09 & 58,8 & 111,7 \\
\hline $\begin{array}{l}\mathrm{KCl}-\mathrm{NaCl}-\mathrm{SrCl}_{2} \\
19,2 \quad 34,9 \quad 45,9\end{array}$ & 6,34 & 6,62 & 3,88 & $-1,02$ & 2,34 & 777 & 107,5 & 4,29 & 39,9 & 93,4 \\
\hline $\begin{array}{l}\mathrm{KCl}-\mathrm{NaCl}-\mathrm{BaCl}_{2} \\
39,733,3 \quad 27\end{array}$ & 6,34 & 6,62 & 8,0 & $-0,61$ & 2,40 & 815 & 105,3 & 6,27 & 59,5 & 142,9 \\
\hline $\begin{array}{l}\mathrm{KCl}-\mathrm{BaCl}_{2}-\mathrm{CaCl}_{2} \\
39,628,1 \quad 32,3\end{array}$ & 6,34 & 8,0 & 6,78 & $-3,09$ & 2,45 & 824 & 123,9 & 3,86 & 31,2 & 76,33 \\
\hline $\begin{array}{l}\mathrm{K}_{2} \mathrm{CO}_{3}-\mathrm{Na}_{2} \mathrm{CO}_{3}-\mathrm{CaCO}_{3} \\
40,8 \quad 33,3 \quad 25,9\end{array}$ & 6,6 & 6,7 & $(6,6)$ & $-2,07$ & 2,13 & 973 & 118,1 & 4,60 & 39,0 & 83,0 \\
\hline
\end{tabular}

${ }^{*}$ ) Densité du mélange liquide au point de fusion.

$\left[\left(^{*}\right)\right.$ Liquid density at melting point.]

ressant de retenir le mélange eutectique binaire dont les constituants purs ont les températures de fusion les plus élevées? pour tenter d'apporter une réponse à ce problème, nous avons calculé pour chacun des mélanges du tableau II la valeur du paramètre $\varepsilon$ défini empiriquement par :

$\varepsilon=X_{\mathrm{A}}^{\mathrm{E}} X_{\mathrm{B}}^{\mathrm{E}}\left[\left(T_{\mathrm{A}}-T_{\mathrm{E}}\right)+\left(T_{\mathrm{B}}-T_{\mathrm{E}}\right)\right]$

relation qui prend en compte la composition $X_{\mathrm{A}}^{\mathrm{E}} \mathrm{du}$ mélange eutectique et les écarts entre la température eutectique $T_{\mathrm{E}}$ et les températures de fusion $T_{\mathrm{A}}$ et $T_{\mathrm{B}}$ des constituants purs. Une corrélation est difficile à établir entre l'enthalpie de fusion du mélange eutectique et ce paramètre; toutefois autour de la température $773 \mathrm{~K}$ plusieurs mélanges eutectiques peuvent être ainsi comparés : il semble que celui qui présente le paramètre $\varepsilon$ le plus grand possède aussi la meilleure enthalpie de fusion (Fig. 2).

4. Conclusion. - A l'aide du calcul thermodynamique exposé ci-dessus et connaissant les enthalpies de fusion des corps purs, il est possible d'évaluer l'enthalpie de fusion d'un mélange eutectique. Mais, avec les hypothèses qui se sont révélées indispensables lors de l'application de la relation proposée, une incertitude de l'ordre de 10 à $15 \%$ n'est pas à exclure, incertitude qui pourrait être gênante dans l'établissement d'un projet de stockage thermique de l'énergie mettant en jeu de grandes quantités de sels. Ce travail est donc une approche qui doit permettre un

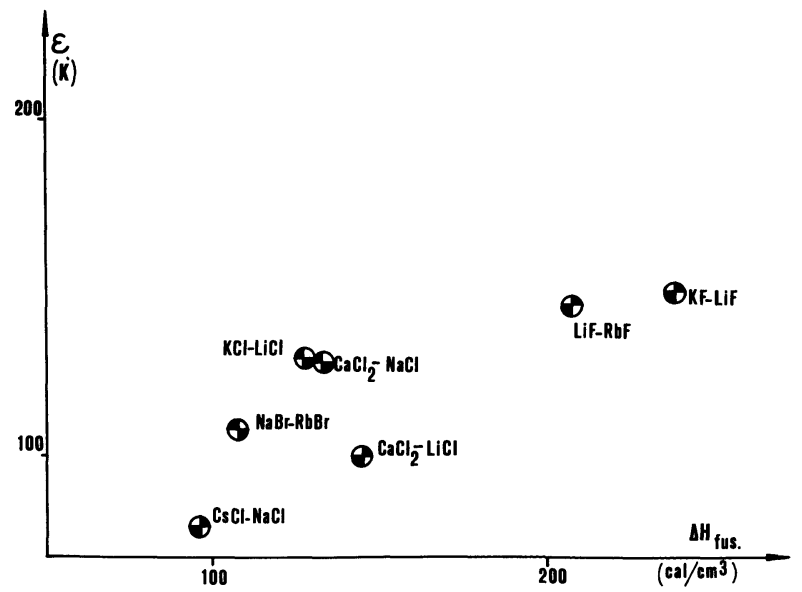

Fig. 2. - Variation de $\varepsilon\left({ }^{*}\right)$ en fonction de l'enthalpie de fusion $\Delta H_{\text {fus. }}^{\mathrm{E}}\left(\mathrm{cal} / \mathrm{cm}^{3}\right)$ de mélanges eutectiques binaires de sels fondus $\left(740 \mathrm{~K}<T^{\mathrm{E}}<780 \mathrm{~K}\right)$.

$\left(^{*}\right)$ Les concentrations $x_{\mathrm{A}}^{\mathrm{E}}$ et $x_{\mathrm{B}}^{\mathrm{E}}$ intervenant dans la relation de définition de $\varepsilon$ ont été rapportées, par souci d'homogénéité, à une base volumique (fractions volumiques).

[Plot of $\varepsilon\left({ }^{*}\right)$ against the enthalpy of fusion $\Delta H_{\mathrm{fus} .}^{\mathrm{E}}\left(\mathrm{cal} / \mathrm{cm}^{3}\right)$ of binary eutectic molten salt mixtures $\left(740 \mathrm{~K}<T^{\mathrm{E}}<780 \mathrm{~K}\right)$.]

$\left[\left(^{*}\right)\right.$ The concentrations $x_{\mathrm{A}}^{\mathrm{E}}$ and $x_{\mathrm{B}}^{\mathrm{E}}$ in the relationship giving $\varepsilon$ has been referred, for homogeneity sake, to volume scale (volume fractions).]

premier choix parmi les systèmes eutectiques; il n'exclut en aucun cas les déterminations expérimentales des enthalpies de fusion. 


\section{Bibliographie}

[1] Table Ronde Stockage thermique de l'énergie, Marseille (1976). Table Ronde Stockage thermique de l'énergie, Chatou (1977). Table Ronde Stockage thermique de l'énergie, Marseille (1978).

[2] BonNin, J., Rapport EDF/DER J30/73-15 (1973).

[3] Bonnin, J., Rapport EDF no P00/76.1 (1976).

[4] Rollet, A: P., Bouazis, R. (Gauthier-Villars, Paris) 1972.

[5] Fouque, Y., Gaune-Escard, M., Sczepaniak, W., Bogacz, A., J. Chim. Phys., no 4 (1978) 360-366.

[6] Bros, J. P., Thèse Doctorat Sci. Phys., Marseille (1968).

[7] KubacheWsKI, O., La thermochimie en métallurgie (GauthierVillars, Paris) 1964.

[8] Gaune-EsCard, M., Thèse Université Aix-Marseille, Marseille (1972).

[9] BLANDER, M., Molten salt chemistry (Interscience Publishers N.Y.) 1964.

[10] Gaune-Escard, M., J. Chim. Phys., no 9 (1974) 1167-1174. J. Chim. Phys., no 9 (1974) 1175-1179.

[11] Voskresenskaya, N. K., Handbook of solid-liquid equilibria in systems of anhydrous Inorganic Salts (Izdatel'Stvo Akademii Nauk SSSR, Moscou-Leningrad) 1961, vol. I et II.
[12] Levin, E. M., Robbins, C. R., McMurdie, H. F., Phase diagrams for ceramists (American Ceramic Society Inc.) 1964. Supplements (1969). Supplements (1975).

[13] JANZ, G. J., Molten Salt Handbook (Academic Press, N.Y., London) 1967.

[14] Janz, G. J., Dampier, F. W., Lakshminarayanan, G. R., Lorenz, P. K., Tomkins, R. P. T., Nat. Stand. Ref. Data Ser., NBS (U.S.) 15 (1968) 1-139.

[15] Janz, G. J., KRebs, U., SiegenthaleR, H. F., Tomkins, R. P. T., J. Phys. Chem. Ref. Data 1 (1972) 581-476.

[16] Janz, G. J., Gardner, G. L., Krebs, U., Tomkins, R. P. T., J. Phys. Chem. Ref. Data 3 (1974) 1-116.

[17] Janz, G. J., Tomkins, R. P. T., Allens, C. B., Downey, J. R., SINGer, S. K., J. Phys. Chem. Ref. Data 6 (1977) 409-596.

[18] Lumsden, J., Thermodynamics of Molten Salts Mixtures (Academic Press, N.Y., London) 1966.

[19] ØstvoLD, T., Thèse, University of Trondheim, NTH (Norway) 1971.

[20] Richardson, F. D., Physical Chemistry of Melts in Metallurgy, Vol. I (Academic Press, N.Y., London) 1974, Vol. I. 